\title{
Combinación entre Algoritmos Genéticos y Aleatorios para la Programación de Horarios de Clases basado en Ritmos Cognitivos
}

\author{
Omar D. Castrillón \\ Universidad Nacional de Colombia, Departamento de Ingeniería Industrial, Campus la Nubia, \\ Manizales-Colombia (e-mail: odcastrillong@unal.edu.co)
}

Recibido Ene. 8, 2014; Aceptado Feb. 12, 2014; Versión final recibida Mar. 15, 2014

\begin{abstract}
Resumen
Se ha diseñado un método basado en algoritmos evolutivos (genéticos y aleatorios) para programar los horarios de clases en una universidad. Esta metodología considera los ritmos cognitivos de los estudiantes que indican que es mejor enseñar algunas asignaturas en intervalos específicos de tiempo. Primero se describen las diferentes técnicas empleadas para desarrollar este problema. Luego se propone una nueva metodología basada en ritmos cognitivos y algoritmos evolutivos, para resolver todas las restricciones duras y blandas del problema. Finalmente se comparan diferentes metodologías para establecer cuál es la más eficiente. Se concluye que los algoritmos evolutivos son más eficientes que otras técnicas empleadas $(19.5 \%)$ en la programación de horarios universitarios.
\end{abstract}

Palabras clave: programación, horarios universitarios, algoritmos genéticos, función objetivo, restricciones

\section{Combination Between Genetic and Random Algorithms for Programing Class Schedule based on Cognitive Rhythms}

\begin{abstract}
A method based on evolutionary algorithms (genetics and random) to plan class schedules in a university has been developed. This methodology considers the students' cognitive rhythms, which indicate that it is better to teach some subjects in some specific time intervals. First, the different techniques to solve this problem are described. Then, a new methodology based on cognitive rhythms and evolutionary algorithms is proposed, to solve the hard and soft constraints. Finally, different methodologies are compared to determine the most efficient one. It is concluded that evolutionary algorithms are more efficient than others techniques $(19.5 \%)$ for planning class schedules.
\end{abstract}

Keywords: scheduling, university timetables, genetic algorithms, fitness, hard and soft constraints. 


\section{INTRODUCCIÓN}

Hoy en día han sido desarrolladas muchas técnicas en la programación de horarios universitarios (Kahar, M y Kendall, 2010; Mejía y Paternina 2010,) sin embargo estas metodologías no consideran los ritmos cognitivos de los estudiantes los cuales permiten determinar el mejor momento en que una asignatura debe ser impartida (Martínez et al 2004; Valentinuzzi y Fontenele, 2008; Suarez et al 2013a; Suarez et al 2013b) En general, las asignaturas con un alto grado de dificultad deben ser enseñadas en la mitad de la jornada escolar, las asignaturas de dificultad moderada deben ser enseñadas al inicio de la jornada escolar, mientras que las asignaturas más fáciles deben ser enseñadas el final de la jornada escolar.

El problema de programación de horarios, en los últimos años ha cobrado especial interés en las Universidades como se muestra en la siguiente revisión literaria: En Mejía y Paternina (2010) se busca resolver el problema de horarios universitarios mediante una meta-heurística basada en algoritmos evolutivos, para un programa de ingeniería específico, considerando que existen asignaciones previas para que algunas materias sean dictadas en periodos especiales. Igualmente modelos similares para universidades colombianas son mostrados en Franco et al (2008), Granada et al, (2006), Caballero et al (2004). En general, este problema en las Universidades debe considerar aspectos como: periodos de tiempo, disponibilidad de salones, disponibilidad docente, cruce de horarios en un mismo semestre, subperiodos de tiempo de un profesor en una asignatura, (Hernández y Miranda 2008), (Abdullah y Turabieh, 2012). Así mismo, los ritmos cognitivos están directamente asociados a los ritmos circadianos, los cuales incluyen factores como: complejidad, diferencias individuales (sexo, cronotipo, estilo, motivación) factores urbanos y rurales (Suarez, 2012). Aspectos muy marcados en las Universidades Colombianas por ende, la aplicación de estos ritmos cognitivos también cobra especial importancia en el contexto universitario.

Básicamente en este documento se presentan dos formas para programar los horarios: a) Basadas en inteligencia artificial b) basadas en métodos tradicionales (prueba y error). Las técnicas basadas en inteligencia artificial permiten obtener buenas soluciones en tan solo unos segundos (Suarez et al 2013b; Suarez et al 2013b; Suarez y Castrillón 2011; Castrillón 2013). Una revisión literaria de los últimos 20 años, permite ilustrar diversas metodologías para la programación de horarios universitarios: En 1992, Cangaiovic y Schreuder (1992) consideraron que no existía un modelo universal para resolver esta clase de problemas, clasificando los problemas en tres grupos fundamentales: profesores, horarios y exámenes. Cuatro más años más adelante, en 1996 se encuentran los trabajos de Boizumault y Delon (1996) quienes resolvieron este problema por medio de una programación lógica de restricción en un domino finito.

Igualmente, Mausser y Magazine (1996) compararon una red neuronal y un heurístico para la solución de este problema. En este mismo año, Gunadhi et al (1996) resolvieron este problema por medio de programación orientada a objetos. Un año más tarde, Thompson y Dowsland (1998), solucionaron el problema de programación de horarios universitarios por medio de simulación anidada. En 1999, Deris et al (1999) propusieron un algoritmo hibrido para solucionar este problema. En el año 2000, (Deris et al 2000) diseñaron una solución basada en definiciones combinatorias y algoritmos dinámicos. En 2002, Asratian y Werra (2002), formularon un nuevo modelo matemático con algunas nuevas restricciones y propiedades, considerando un problema general con $\mathrm{N}$ profesores y $\mathrm{M}$ materias.

En el 2003 una red Hopfiel es propuesta por Smith et al (2003) para solucionar este problema, como resultado se definieron una nueva metodología tan eficiente como las meta heurísticas existentes. En el 2004, Abbas y Tsang (2004) consideraron algunas restricciones nuevas en la solución de este problema (Los estudiantes debían ser pre registrados). En el año 2005, Naji y Azimi (2005) analizaron cuatro técnicas y tres algoritmos híbridos para solucionar este problema, concluyendo que las metodologías hibridas son mejores en la solución de este clase de problemas. En el 2006 Dammak et al (2006) establecieron una solución basada en programación entera y el problema del transporte. En el 2007, Al Yakoob y Sherali (2007), Diseñan un problema de programación de horarios basado en las idiosincrasias de cada problema bajo un problemas de programación matemática. Similarmente (Boland et al 2008), soluciona este problema con un modelo de programación lineal entera, bajo un método llamado bloqueo. finalmente en este año Pongcharoen et al (2008), proponen un modelo de programación estocástica.

Un año más tarde en el 2009, Aladag et al (2009) presentaron un algoritmo basado en una estructura de vecindario para solucionar este problema, así mismo Azmi y Tajudin (2009), definieron un algoritmo hibrido basado en algoritmos armónicos y optimizadores escaladores. En el 2010, Abdullah et al (2010) desarrollaron una metaheristica basada en conferencias y tiempos para solucionar las restricciones duras y minimizar las blandas. Así mismo Berghammer y Kehden (2010), describieron este problema como una relación entre clases e intervalos de tiempo. Burke et al (2010a), codificaron un algoritmo hibrido basado en algoritmos genéticos y un vecindario de búsqueda. Igualmente en este año se encuentran autores como Gogos et al (2010), quienes diseñaron un algoritmo de búsqueda adaptativa y codiciosa combinado varias 
meta-heurísticas para solucionar este problema. Por ultimo en este año, en Kahar y Kendall (2010), se propusieron algunas restricciones nuevas referentes a exámenes y partición de exámenes.

Un año más tarde, Ayob et al (2011) solucionaron el problema en dos fases (solución de restricciones duras y blandas). En este mismo año Hao y Benlic (2011), crearon una metodología basada en la técnica divide y conquistaras, por su parte Wu (2011) diseñó un sistema experto para solución de este problema, igualmente Abdullah y Turabieh (2012), propusieron un algoritmo de búsqueda tabú con tres estrategias diferentes para seleccionar el vecindario: aleatoria, mejor, general. Así mismo en Ceschia et al (2012), solucionaron este problema considerando el pos enrolamiento de los estudiantes bajo un método de simulación anidada.

En forma similar, Gunawan y Poh (2012) escribieron un algoritmo basado en el método de relajación de LaGrange y simulación anidada. Más tarde en el 2013, un algoritmo basado en colonia de hormigas es propuesto por Thepphakorn et al (2013), así mismo en Suarez et al (2013a) y Suarez et al (2013b) se soluciona este problema para un colegio basado en el método NSGA II y un algoritmo genético respectivamente, siendo estos los únicos trabajos que consideran los ritmos cognitivos de los estudiantes, sin embargo el problema solo se resuelve para secundaria. Finalmente, otros autores como Qaurooni et al (2013) emplearon un algoritmo basado en operadores evolutivos. En el 2014, Abdul Rahman et al (2014) construyeron una combinación lineal adaptativa entre coloreo de grafos y múltiples heurísticas, en la solución de estas clase de problemas.

En resumen, en la figura 1, se ilustran las principales técnicas empleadas en los últimos 20 años en la programación de horarios. Entre estas técnicas se tienen: Heurísticos, modelos matemáticos, búsqueda tabú, programación entera, algoritmos evolutivos, algoritmos genéticos, redes neuronales, optimización estocástica, meta-heurísticos y híper-heurísticos, programación orientada a objetos, programación con restricciones, colonia de hormigas, programación matemática, bases de conocimiento, programación lineal, programación lógica, algoritmos aleatorios, algoritmos de abejas, coloreo de grafos, partículas inteligentes, algoritmos híbridos y mímicos, entre otros. En términos generales, este problema en la Universidades ha sido ampliamente estudiando cómo se ilustra en la extensiva revisión literaria que se realiza, sin embargo es importante resaltar que el mismo no ha sido solucionado incluyendo la programación de los ritmos cognitivos de los estudiantes, lo cual implicaría una serie de nueva restricciones blandas para ser consideradas en la solución del problema.

En este artículo se diseña una nueva metodología para la programación de los horarios universitarios basados en el aprovechamiento de los ritmos cognitivos de los estudiantes. En comparación con las técnicas tradicionales, se concluye que esta metodología es por lo menos un $19.5 \%$ más eficiente que las técnicas tradicionales en el aprovechamiento de estos ritmos.

\section{METODOLOGÍA}

Para el desarrollo de este trabajo se propone una metodología basada en dos algoritmos evolutivos y aleatorios, como se describe en los siguientes pasos:

Descripción del problema: En la solución del problema se emplean 4 multidimensionales arreglos: Datos, horarios, profesores y salones. Las tablas 1 y la figura 2 ilustran este aspecto:

Tabla 1: Definición del problema

\begin{tabular}{|c|c|c|c|c|c|c|c|c|c|c|c|}
\hline Sem & $\begin{array}{c}\text { Código } \\
\text { Materia }\end{array}$ & Nombre & $\begin{array}{c}\text { Horas/ } \\
\text { Semana }\end{array}$ & Gr & $\begin{array}{c}\text { Prof } \\
\text { Cod }\end{array}$ & $\begin{array}{c}\text { Prof } \\
\text { Nom }\end{array}$ & Max/Hor/Dia & Salón & $\begin{array}{c}\text { Hora } \\
\text { Ideal }\end{array}$ & $\begin{array}{c}\text { Prof_2 } \\
\text { Cod }\end{array}$ & $\begin{array}{c}\text { Prof_2 } \\
\text { Nom }\end{array}$ \\
\hline 1 & 1030 & Física & 4 & 1 & 1 & $\begin{array}{c}\text { Prof. } \\
1\end{array}$ & 2 & 1 & 10 & 2 & Prof. 2 \\
\hline$\ldots$ &.. & $\ldots$ & $\ldots$ &.. &.. & $\ldots$ & $\ldots$ & $\ldots$ & $\ldots$ & $\ldots$ & $\ldots$ \\
\hline 10 & 1562 & Proyecto & 2 & 2 & 1 & $\begin{array}{c}\text { Prof. } \\
3\end{array}$ & 2 & 3 & 14 & & \\
\hline
\end{tabular}




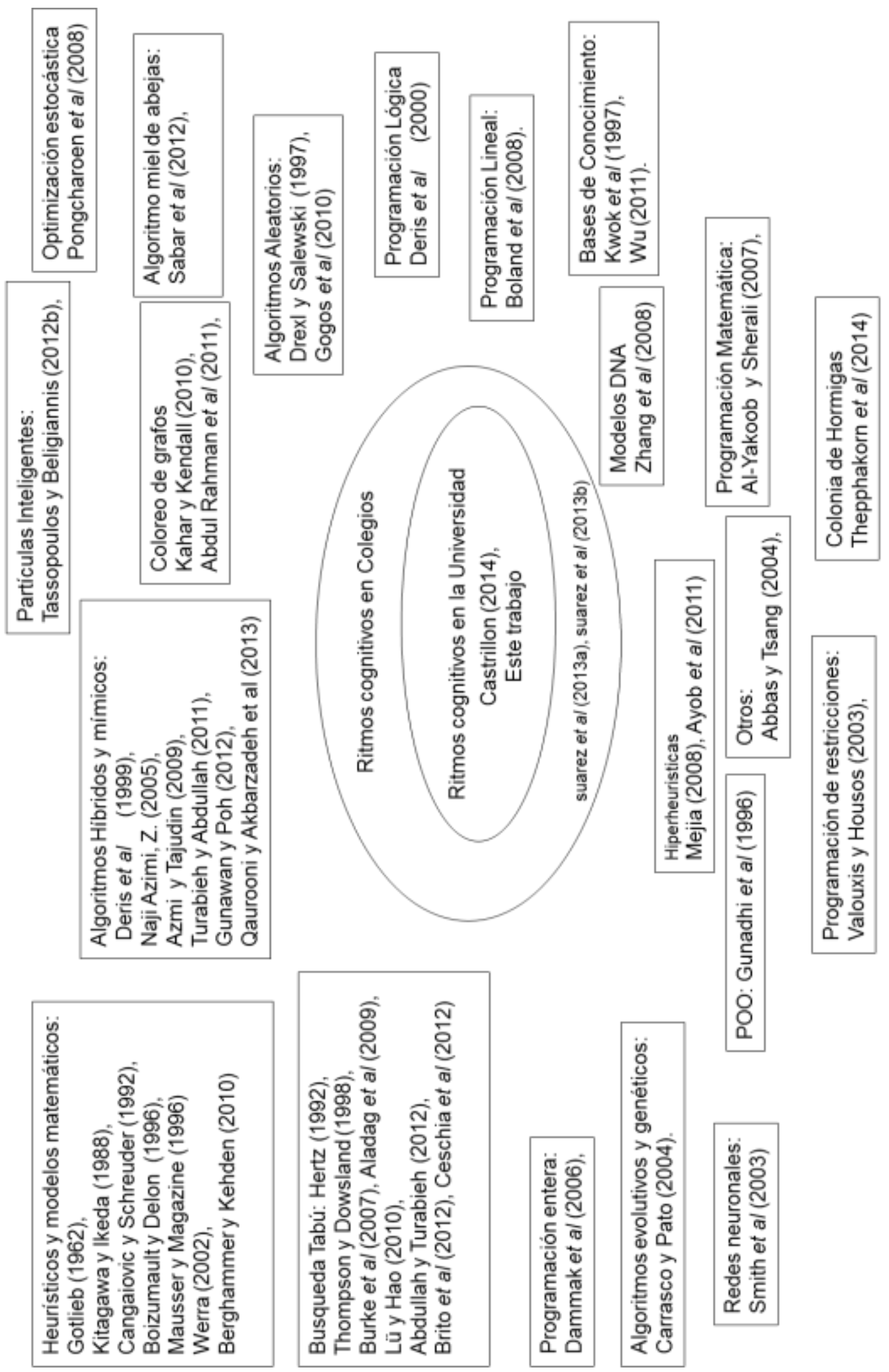

Fig. 1. Programación de horarios universitarios. 
En la tabla 1, se ilustran doce campos de derecha a izquierda: Semestre, código de la materia, nombre de la materia, horas por semana, grupo, código del profesor, máximo horas por día, salón, hora ideal, código profesor auxiliar, nombre profesor auxiliar. En el problema son consideradas algunas restricciones duras y blandas. Entre las restricciones duras se encuentras: a) número de profesores y asignación. b) tipo de salón (normal, informática o audiovisual). c) máximo de horas por día. d) todas las materias de un mismo semestre deben ser programas en diferentes horas. e) dos o más materias no pueden ser atendidas por el mismo profesor al mismo tiempo. f) dos o más materias no pueden ser programadas en el mismo salón. g) los profesores y salones pueden tener algunos periodos en los cuales no se encuentran disponibles. $h$ ) dos o más horas de una misma materia deben estar seguidas. Igualmente se deben satisfacer las siguientes restricciones blandas: a) Las materias deben ser programadas en la hora ideal. El mejor momento para enseñar cada asignatura es dado por los ritmos cognitivos de los estudiantes. b) el horario debe ser tan compacto como sea posible (menor cantidad de horas libres entre la primera hora y la última del día). La estructura tridimensional ilustrada en la figura 1, muestra la definición de datos empleada para solucionar las restricciones. Las restricciones duras tiene que ser resultas mientras que las blandas deben ser optimizadas. (Se emplean tres estructuras diferentes para salones, semestres y profesores)

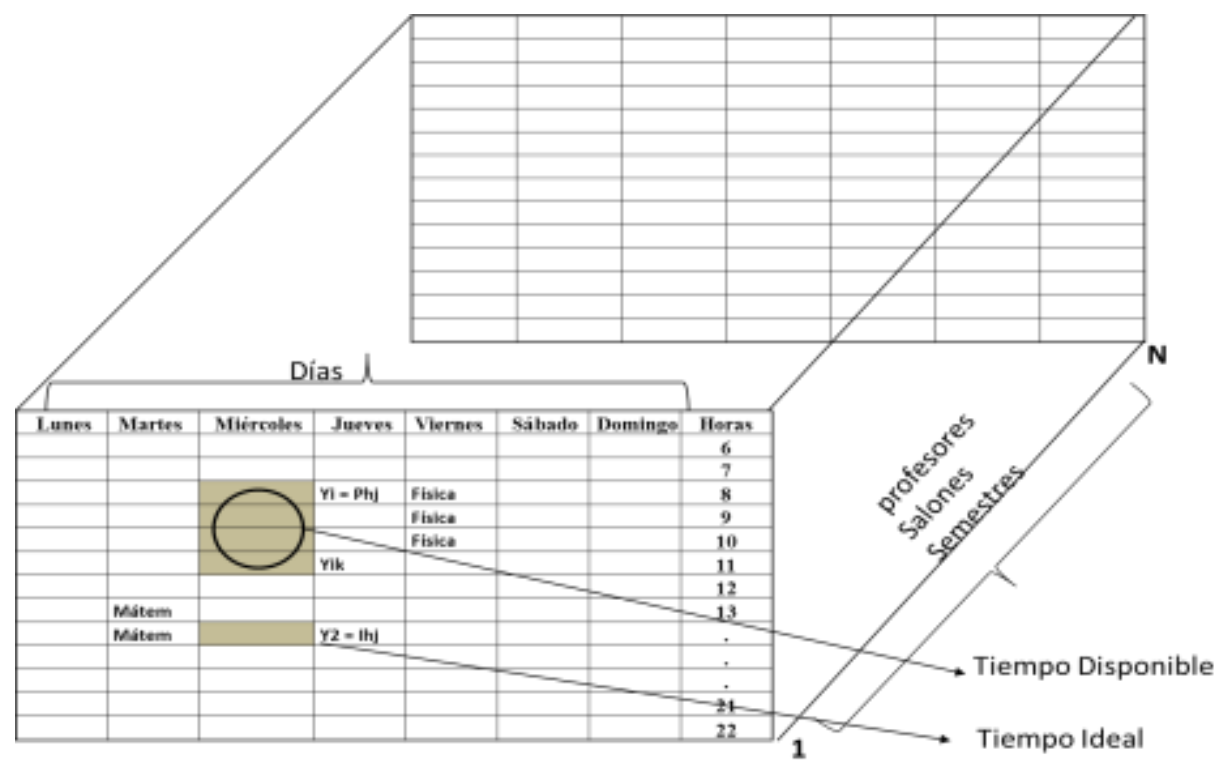

Fig. 2. Estructura de datos empleada y penalidad $=\boldsymbol{a} \boldsymbol{b} \boldsymbol{s}\left(\boldsymbol{I} \boldsymbol{h}_{\boldsymbol{j}}-\boldsymbol{P} \boldsymbol{h}_{\boldsymbol{j}}\right)$

Tomando como referencia un enfoque basado en programación lógica, podemos emplear la siguiente codificación para resolver las restricciones duras y optimizar las restricciones blandas:

$\mathrm{x}=$ Dias $($ Range $=1 . .6)$

$\mathrm{y}=$ Horas $($ Range $=1 \ldots 15)$

$\mathrm{z} 1=$ Profesores $($ Range $=1 \ldots \mathrm{Np})$

$\mathrm{z} 2=$ Semestre $($ Range $=1 \ldots \mathrm{Ns})$

$\mathrm{z} 3=$ Salones $($ Range $=1 \ldots \mathrm{Nr})$

Map $=$ Horario de profesores (Arreglo Multidimensional)

Mas $=$ Horario de semestres (Arreglo Multidimensional)

Mar $=$ Horario de salones (Arreglo Multidimensional)

$\mathrm{k}$ = Máximo de horas por material / Día.

$\mathrm{lh}=$ Tiempo ideal de la materia

$\mathrm{Ph}$ Tiempo asignado a la material.

Se deben encontrar las variables $x, y, z$ tales que:

Si $\quad\left(\left(\operatorname{Map}\left(x, y_{i . .} y_{i k}, z 1\right)=[\right.\right.$ Disponible] $)$ y $\left(\operatorname{Mas}\left(\mathrm{x}, y_{i . .} y_{i k}, z 2\right)=[\right.$ Disponible] $) y$ $\left(\operatorname{Mar}\left(\mathrm{x}, y_{i} . y_{i k}, \mathrm{z} 3\right)=[\right.$ Disponible] $\left.)\right)$ entonces (Los periodos estan disponibles).

$\operatorname{Map}\left(x, y_{i . .} y_{i k}, z 1\right)=[$ La asignatura es programada $]$

$\operatorname{Mas}\left(\mathrm{x}, y_{i . . .} y_{i k}, \mathrm{z} 2\right)=[$ La asignatura es programada $]$

$\operatorname{Mar}\left(\mathrm{x}, y_{i} . y_{i k}, \mathrm{z} 3\right)=[$ La asignatura es programada $]$

Penalidad (Figura 1)

Else

End

Solución Infactible 
Nota: Aunque la solución total del problema presenta más de 5000 líneas de código, se considera importante las anteriores 20 líneas de código dado que las mismas formalizan la resolución de restricciones duras y optimización de las blandas.

Para las soluciones factibles se define la siguiente penalidad por cada asignatura programada: Penalidad $=\operatorname{abs}\left(I h_{j}-P h_{j}\right)$.

Descripción del algoritmo:

Paso 1: Población Inicial: cada una de las asignaturas representadas en la tablas 1, son programadas dentro de las tres estructuras tridimensionales, según la secuencia que se establezca en la tabla 2, esta secuencia se conoce como gen inicial.

Tabla 2: Secuencia de programación (Padre Inicial)

\begin{tabular}{|l|l|l|l|l|l|l|l|}
\hline $1 . .$. & (Rows number of table 1 = N) & & \\
\hline & & & & & & & \\
\hline
\end{tabular}

Paso 2: Operador aleatorio: Por medio de este operador se genera un nuevo padre de forma aleatoria. Según la estructura de la tabla 2. Esta generación aleatoria se emplea cuando el algoritmo ha caído en óptimo local. Estancamiento ( $\mathrm{K}$ veces sin encontrar una mejorar solución), este táctica permitirá explorar nuevas rutas de evolución. Con el fin de verificar que la solución sea válida, es necesario construir el horario representado por el nuevo padre, en caso contrario se continuará generando nuevos padres hasta encontrar uno que contenga una solución válida. Una vez encontrado un padre valido, se volverá a iniciar un proceso evolutivo del mismo, el cual permita mejorar esta solución.

Pasó 3: Operador de mutación: Este operador cambia dos posiciones definidas aleatoriamente en la tabla 2. Ocurre con un tres por ciento de probabilidad.

Paso 4: Operador de combinación. Este operador combina dos diferentes padres para generar un nuevo padre. El mismo ocurre con un $97 \%$ de probabilidad. El proceso de combinación se realiza sobre un tercer vector. Inicialmente las $\mathrm{k}$ últimas posiciones del padre dos, se copian sobre las k primeras posiciones del nuevo padre. Posteriormente se realiza un recorrido sobre el padre uno, para determinar los números que se encuentran en el padre uno, que no están en el nuevo padre e insertarlos en este último vector, según el orden que aparezcan en el padre uno. Es probable que la nueva solucione generada mediante los operadores de mutación y combinación en un momento determinado no representen una solución válida, en este caso se graba la mejor solución encontrada hasta el momento y se inicia un nuevo proceso evolutivo con otro padre valido.

Paso 5: Empleo de los operadores. Este algoritmo inicia una evolución por medio de los algoritmos genéticos, cuando el operador se estanca en un óptimo local, se genera un padre aleatoriamente con el fin de introducir nuevas variantes que permitan continuar con la evolución del problema.

Paso 6: Condición de parada. El algoritmo finaliza cuando alcanza un óptimo global (Penalidad total $=0)$ o cuando evoluciona un determinado número de veces sin superar la mejor solución encontrada.

Paso 7: Fitness. Según lo ilustrado en la figura 1, una función de penalidad es definida por cada asignatura como la diferencia entre la hora ideal y la hora programada. La sumatoria de las penalidades es definida como la función Fitness.

Fitness $=\sum_{J=1}^{\text {Asignaturas }} A b s\left(I h_{j}-P h_{j}\right)$

Los horarios generados por semestre deben ser lo menos dispersos posibles (compactos) es decir deben tener el menor número de horas libres entre la primera asignatura programada en un día en el semestre y la última asignatura programada en ese mismo día en ese semestre, la suma de los índices diarios es el índice semanal. Para calcular este índice, primero se calculan las soluciones que cumplan las restricciones duras y blandas y posteriormente se genera este índice recorriendo la estructura tridimensional semestre.

Paso 8. Análisis Estadístico: La soluciones generadas son repetidas durante determinada cantidad de veces ó tratamientos. Los 10 mejores resultados de la función fitness son seleccionados en cada tratamiento. Usando estos resultados un análisis de varianza bajo el modelo $y_{i}=\mu+T_{i}+\varepsilon_{i}$, donde $y_{i}$ representa la variable de la respuesta, $\mathrm{T}_{\mathrm{i}}$, el efecto causado por $i^{\text {th }}$ tratamiento, $\varepsilon$ i, el $i^{\text {th }}$ error experimental. La información recolectada debe cumplir las condiciones de independencia y normalidad requeridas. 
Paso 9: Comparación. Los mejores resultados obtenidos en esta metodología son comparados con los resultados obtenidos bajo técnicas tradicionales o aquellas basadas en procesos de prueba y error en la solución de este problema. En la programación tradicional las asignaturas son programadas en el orden inicial en que son definidas (tabla 1) sin que este orden sea variado. Solo existe un resultado.

\section{Experimentación}

En la tabla 3 se ilustra el problema que será solucionado, el cual es definido según la estructura de la tabla 1. Por razones de espacio este problema se limita a 18 asignaturas y tres semestres. Sin embargo el mismo es un problema NP-Completo el cual presenta $18 !=6.40^{*} 10^{15}$ posibilidades de programación. Este problema se programa sobre una disponibilidad diaria de 15 horas entre 7:00 a.m. y 21:00 p.m., considerando cuatro salones. Dos salones tipo 1 (aula normal y numerados como 1 y 2), un salón tipo 2 (aula audio visual, numerado como 3) y un salón tipo 3 (aula informática, numerado como 4). Igualmente se considerará que el profesor IO (código 7) no puede trabajar el día martes, después de las 2:00 P.M.

Además el salón tipo 1 (numerado como 1) no está disponible los miércoles por las mañanas. Para tratar de programar las asignaturas en la hora ideal es importante que los horarios por semestre sean lo menos dispersos posibles (menor número de horas libres entre la primera asignatura programada en un día y la última asignatura programada en el mismo día en el mismo semestre). La hora ideal para cada asignatura, se determinó según trabajos previos (Suarez 2012).

Tabla 3: Problema Objeto de estudio. $18 !=6.40^{*} 10^{15}$ posibilidades

\begin{tabular}{|l|l|l|l|l|l|l|l|l|l|l|l|}
\hline Sem & $\begin{array}{l}\text { Código } \\
\text { Materia }\end{array}$ & Nombre & $\begin{array}{l}\text { Horas/ } \\
\text { Semana }\end{array}$ & Gr & Prof. & Prof. & $\begin{array}{l}\text { Max/Hor } \\
\text { /Día }\end{array}$ & Salón & $\begin{array}{l}\text { Hora } \\
\text { Ideal }\end{array}$ & $\begin{array}{l}\text { Prof_2 } \\
\text { Código }\end{array}$ & $\begin{array}{l}\text { Prof_2 } \\
\text { Nome }\end{array}$ \\
\hline 4 & 14 & OPTA-MATEMA & 4 & 1 & 1 & Prof 1 & 2 & 1 & 9 & 0 & \\
\hline 4 & 15 & OFI EMPRE & 4 & 1 & 2 & Prof 2 & 2 & 3 & 9 & 0 & \\
\hline 4 & 15 & OFI EMPRE & 4 & 2 & 2 & Prof 2 & 2 & 3 & 9 & 0 & \\
\hline 4 & 16 & T MATER & 8 & 1 & 3 & Prof 3 & 3 & 2 & 7 & 0 & \\
\hline 4 & 16 & T MATER & 8 & 2 & 4 & Prof 4 & 3 & 2 & 7 & 0 & \\
\hline 4 & 17 & OPTA-FISICA & 4 & 1 & 5 & Prof 5 & 2 & 1 & 9 & 0 & \\
\hline 5 & 18 & ESTADIS 1 & 4 & 1 & 6 & Prof 6 & 2 & 1 & 9 & 0 & \\
\hline 5 & 19 & IO 1 & 4 & 1 & 7 & Prof 7 & 2 & 1 & 9 & 0 & \\
\hline 5 & 20 & $\begin{array}{l}\text { TALLER DE } \\
\text { PROCESOS }\end{array}$ & 10 & 1 & 8 & Prof 8 & 3 & 2 & 7 & 7 & Prof 7 \\
\hline 5 & 20 & $\begin{array}{l}\text { TALLER DE } \\
\text { PROCESOS }\end{array}$ & 10 & 2 & 4 & Prof 4 & 3 & 2 & 7 & 0 & \\
\hline 5 & 22 & $\begin{array}{l}\text { OPTATIVA- } \\
\text { MATERIALES }\end{array}$ & 4 & 1 & 3 & Prof 3 & 2 & 1 & 9 & 0 & \\
\hline 5 & 23 & $\begin{array}{l}\text { SISTEMAS DE } \\
\text { COSTOS }\end{array}$ & 6 & 1 & 9 & Prof 9 & 3 & 1 & 16 & 0 & \\
\hline 6 & 24 & ESTADISTICA 2 & 4 & 1 & 10 & prof 10 & 2 & 1 & 9 & 0 & \\
\hline 6 & 25 & IO 2 & 4 & 1 & 7 & Prof 7 & 2 & 1 & 9 & 0 & \\
\hline 6 & 26 & $\begin{array}{l}\text { TALLER DE } \\
\text { METODOS }\end{array}$ & 10 & 1 & 11 & Prof 11 & 5 & 2 & 7 & 0 & \\
\hline 6 & 26 & $\begin{array}{l}\text { TALLER DE } \\
\text { METODOS }\end{array}$ & 10 & 2 & 11 & Prof 11 & 3 & 2 & 7 & 0 & \\
\hline 6 & 27 & H YS INDUSTRIAL & 4 & 1 & 12 & Prof 12 & 2 & 1 & 7 & 0 & \\
\hline 6 & 28 & GESTION HUMANA & 4 & 1 & 13 & Prof 13 & 2 & 1 & 11 & 0 & \\
\hline
\end{tabular}




\section{RESULTADOS}

Como resultado de aplicar la metodología sobre este problema (tabla 2) se encuentra: Paso 1-7. Bajo esta secuencia de pasos se obtiene la siguiente programación de horarios (tablas 4, 5, 6):

Tabla 4: Programación 4 semestre

\begin{tabular}{|c|c|c|c|c|c|}
\hline Hora & Lunes & Martes & Miércoles & Jueves & Viernes \\
\hline 8 & OFI EMPRE-1-AUL:4 & OFI EMPRE-2-AUL:4 & T MATER-1-AUL:3 & T MATER-2-AUL:3 & $\begin{array}{c}\text { T MATER-2- } \\
\text { AUL:3 }\end{array}$ \\
\hline 9 & OFI EMPRE-1-AUL:4 & OFI EMPRE-2-AUL:4 & T MATER-1-AUL:3 & T MATER-2-AUL:3 & $\begin{array}{c}\text { T MATER-2- } \\
\text { AUL: } 3\end{array}$ \\
\hline 10 & OPTA-FISICA-1-AUL:2 & OPTA-MATEMA-1-AUL:2 & $\begin{array}{l}\text { OFI EMPRE-2- } \\
\text { AUL:4 }\end{array}$ & T MATER-1-AUL:3 & $\begin{array}{c}\text { T MATER-2- } \\
\text { AUL:3 }\end{array}$ \\
\hline 11 & OPTA-FISICA-1-AUL:2 & OPTA-MATEMA-1-AUL:2 & $\begin{array}{l}\text { OFI EMPRE-2- } \\
\text { AUL:4 }\end{array}$ & T MATER-1-AUL:3 & $\begin{array}{c}\text { T MATER-1- } \\
\text { AUL:3 }\end{array}$ \\
\hline 12 & OPTA-MATEMA-1-AUL:2 & & $\begin{array}{l}\text { OFI EMPRE-1- } \\
\text { AUL:4 }\end{array}$ & T MATER-1-AUL:3 & $\begin{array}{c}\text { T MATER-1- } \\
\text { AUL:3 }\end{array}$ \\
\hline 13 & OPTA-MATEMA-1-AUL:2 & T MATER-2-AUL:3 & $\begin{array}{l}\text { OFI EMPRE-1- } \\
\text { AUL:4 }\end{array}$ & $\begin{array}{c}\text { OPTA-FISICA-1- } \\
\text { AUL:1 }\end{array}$ & $\begin{array}{c}\text { T MATER-1- } \\
\text { AUL:3 }\end{array}$ \\
\hline \multirow[t]{2}{*}{14} & & T MATER-2-AUL:3 & & $\begin{array}{c}\text { OPTA-FISICA-1- } \\
\text { AUL:1 }\end{array}$ & \\
\hline & & T MATER-2-AUL:3 & & & \\
\hline
\end{tabular}

Tabla 5: Programación 5 semestre.

\begin{tabular}{|c|c|c|c|c|c|}
\hline Hora & Lunes & Martes & Miércoles & Jueves & Viernes \\
\hline 7 & & $\begin{array}{c}\text { TALLER DE } \\
\text { PROCESOS-1-AUL:3 }\end{array}$ & & $\begin{array}{c}\text { TALLER DE } \\
\text { PROCESOS-2-AUL:3 }\end{array}$ & \\
\hline 8 & $\begin{array}{c}\text { TALLER DE } \\
\text { PROCESOS-1-AUL:3 }\end{array}$ & IO 1-1-AUL:1 & & & \\
\hline 9 & $\begin{array}{c}\text { TALLER DE } \\
\text { PROCESOS-1-AUL:3 }\end{array}$ & IO 1-1-AUL:1 & & & $\begin{array}{c}\text { OPTATIVA- } \\
\text { MATERIALES-1-AUL:1 }\end{array}$ \\
\hline 10 & $\begin{array}{c}\text { TALLER DE } \\
\text { PROCESOS-1-AUL:3 }\end{array}$ & $\begin{array}{c}\text { OPTATIVA- } \\
\text { MATERIALES-1-AUL:1 }\end{array}$ & $\begin{array}{c}\text { TALLER DE } \\
\text { PROCESOS-2-AUL:3 }\end{array}$ & ESTADIS 1-1-AUL:1 & $\begin{array}{c}\text { OPTATIVA- } \\
\text { MATERIALES-1-AUL:1 }\end{array}$ \\
\hline 11 & $\begin{array}{c}\text { TALLER DE } \\
\text { PROCESOS-2-AUL:3 }\end{array}$ & $\begin{array}{c}\text { OPTATIVA- } \\
\text { MATERIALES-1-AUL:1 }\end{array}$ & $\begin{array}{c}\text { TALLER DE } \\
\text { PROCESOS-2-AUL:3 }\end{array}$ & ESTADIS 1-1-AUL:1 & IO 1-1-AUL:1 \\
\hline 12 & $\begin{array}{c}\text { TALLER DE } \\
\text { PROCESOS-2-AUL:3 }\end{array}$ & ESTADIS 1-1-AUL:1 & $\begin{array}{c}\text { TALLER DE } \\
\text { PROCESOS-2-AUL:3 }\end{array}$ & & IO 1-1-AUL:1 \\
\hline 13 & $\begin{array}{c}\text { TALLER DE } \\
\text { PROCESOS-2-AUL:3 }\end{array}$ & ESTADIS 1-1-AUL:1 & $\begin{array}{c}\text { TALLER DE } \\
\text { PROCESOS-1-AUL:3 }\end{array}$ & & \\
\hline 14 & & & $\begin{array}{c}\text { TALLER DE } \\
\text { PROCESOS-1-AUL:3 }\end{array}$ & & $\begin{array}{c}\text { TALLER DE } \\
\text { PROCESOS-2-AUL:3 }\end{array}$ \\
\hline 15 & & & $\begin{array}{c}\text { TALLER DE } \\
\text { PROCESOS-1-AUL:3 }\end{array}$ & & $\begin{array}{c}\text { TALLER DE } \\
\text { PROCESOS-2-AUL:3 }\end{array}$ \\
\hline 16 & & & & $\begin{array}{c}\text { TALLER DE } \\
\text { PROCESOS-1-AUL:3 }\end{array}$ & $\begin{array}{c}\text { TALLER DE } \\
\text { PROCESOS-2-AUL:3 }\end{array}$ \\
\hline 17 & $\begin{array}{l}\text { SISTEMAS DE } \\
\text { COSTOS-1-AUL:1 }\end{array}$ & $\begin{array}{l}\text { SISTEMAS DE } \\
\text { COSTOS-1-AUL:1 }\end{array}$ & & $\begin{array}{c}\text { TALLER DE } \\
\text { PROCESOS-1-AUL:3 }\end{array}$ & \\
\hline 18 & $\begin{array}{l}\text { SISTEMAS DE } \\
\text { COSTOS-1-AUL:1 }\end{array}$ & $\begin{array}{l}\text { SISTEMAS DE } \\
\text { COSTOS-1-AUL:1 }\end{array}$ & & $\begin{array}{c}\text { TALLER DE } \\
\text { PROCESOS-1-AUL:3 }\end{array}$ & \\
\hline 19 & $\begin{array}{l}\text { SISTEMAS DE } \\
\text { COSTOS-1-AUL:1 }\end{array}$ & $\begin{array}{l}\text { SISTEMAS DE } \\
\text { COSTOS-1-AUL:1 }\end{array}$ & & & \\
\hline
\end{tabular}


Tabla 6: Programación 6 semestre.

\begin{tabular}{|c|c|c|c|c|c|}
\hline Hora & Lunes & Martes & Miércoles & Jueves & Viernes \\
\hline 7 & & & $\begin{array}{c}\text { TALLER DE } \\
\text { METODOS-2-AUL:3 }\end{array}$ & & \\
\hline 8 & $\begin{array}{c}\text { H Y S INDUSTRIAL-1- } \\
\text { AUL:1 }\end{array}$ & $\begin{array}{c}\text { TALLER DE } \\
\text { METODOS-1-AUL:3 }\end{array}$ & $\begin{array}{c}\text { H Y S INDUSTRIAL-1- } \\
\text { AUL:2 }\end{array}$ & & \\
\hline 9 & $\begin{array}{c}\text { H Y S INDUSTRIAL-1- } \\
\text { AUL:1 }\end{array}$ & $\begin{array}{c}\text { TALLER DE } \\
\text { METODOS-1-AUL:3 }\end{array}$ & $\begin{array}{c}\text { H Y S INDUSTRIAL-1- } \\
\text { AUL:2 }\end{array}$ & & \\
\hline 10 & $\begin{array}{c}\text { ESTADISTICA 2-1- } \\
\text { AUL:1 }\end{array}$ & $\begin{array}{c}\text { TALLER DE } \\
\text { METODOS-1-AUL:3 }\end{array}$ & IO 2-1-AUL:2 & IO 2-1-AUL:2 & $\begin{array}{l}\text { ESTADISTICA 2- } \\
\text { 1-AUL:2 }\end{array}$ \\
\hline 11 & $\begin{array}{l}\text { ESTADISTICA 2-1- } \\
\text { AUL:1 }\end{array}$ & $\begin{array}{c}\text { TALLER DE } \\
\text { METODOS-1-AUL:3 }\end{array}$ & IO 2-1-AUL:2 & IO 2-1-AUL:2 & $\begin{array}{l}\text { ESTADISTICA 2- } \\
\text { 1-AUL:2 }\end{array}$ \\
\hline 12 & $\begin{array}{c}\text { GESTION HUMANA-1- } \\
\text { AUL:1 }\end{array}$ & $\begin{array}{c}\text { TALLER DE } \\
\text { METODOS-1-AUL:3 }\end{array}$ & $\begin{array}{c}\text { GESTION HUMANA-1- } \\
\text { AUL:2 }\end{array}$ & & \\
\hline 13 & $\begin{array}{l}\text { GESTION HUMANA-1- } \\
\text { AUL:1 }\end{array}$ & & $\begin{array}{c}\text { GESTION HUMANA-1- } \\
\text { AUL:2 }\end{array}$ & $\begin{array}{c}\text { TALLER DE } \\
\text { METODOS-2-AUL:3 }\end{array}$ & \\
\hline 14 & $\begin{array}{c}\text { TALLER DE } \\
\text { METODOS-2-AUL:3 }\end{array}$ & & & $\begin{array}{c}\text { TALLER DE } \\
\text { METODOS-2-AUL:3 }\end{array}$ & \\
\hline 15 & $\begin{array}{c}\text { TALLER DE } \\
\text { METODOS-2-AUL:3 }\end{array}$ & & & $\begin{array}{c}\text { TALLER DE } \\
\text { METODOS-2-AUL:3 }\end{array}$ & \\
\hline 16 & $\begin{array}{c}\text { TALLER DE } \\
\text { METODOS-2-AUL:3 }\end{array}$ & $\begin{array}{c}\text { TALLER DE } \\
\text { METODOS-2-AUL:3 }\end{array}$ & $\begin{array}{c}\text { TALLER DE } \\
\text { METODOS-1-AUL:3 }\end{array}$ & & \\
\hline 17 & & $\begin{array}{c}\text { TALLER DE } \\
\text { METODOS-2-AUL:3 }\end{array}$ & $\begin{array}{c}\text { TALLER DE } \\
\text { METODOS-1-AUL:3 }\end{array}$ & & \\
\hline 18 & & $\begin{array}{c}\text { TALLER DE } \\
\text { METODOS-2-AUL:3 }\end{array}$ & $\begin{array}{c}\text { TALLER DE } \\
\text { METODOS-1-AUL:3 }\end{array}$ & & \\
\hline \multirow[t]{2}{*}{19} & & & $\begin{array}{c}\text { TALLER DE } \\
\text { METODOS-1-AUL:3 }\end{array}$ & & \\
\hline & & & $\begin{array}{c}\text { TALLER DE } \\
\text { METODOS-1-AUL:3 }\end{array}$ & & \\
\hline
\end{tabular}

La solución presentada tiene una función fitness de 80. La misma cumple con todas las restricciones duras y optimiza las blandas según lo descrito en la sección uno de la metodología y en la definición del problema.

Paso 8-9: Como resultado de estos pasos se obtiene el siguiente análisis de varianza (Tablas 7 y 8):

Tabla 7: Análisis de varianza. Repetición de datos.

\begin{tabular}{|c|c|c|c|c|c|c|c|c|c|c|c|}
\hline Fitness & \multicolumn{1}{c|}{ Repeticiones } & \multicolumn{1}{c|}{ Sum } \\
\multicolumn{1}{c}{1} & 2 & 3 & 4 & 5 & 6 & 7 & 8 & 9 & 10 & \\
\hline Trata 1 & 80 & 81 & 84 & 82 & 83 & 82 & 85 & 86 & 81 & 82 & 826 \\
\cline { 2 - 11 } Trata 2 & 83 & 82 & 82 & 83 & 84 & 82 & 85 & 81 & 81 & 83 & 826 \\
\cline { 2 - 10 } Trata 3 & 85 & 81 & 81 & 82 & 84 & 83 & 81 & 81 & 81 & 84 & 823 \\
\hline Trata 4 & 82 & 86 & 81 & 83 & 81 & 85 & 82 & 84 & 81 & 81 & 826 \\
\hline
\end{tabular}

Tabla 8: Análisis de varianza. Calculo función $\mathrm{F}$

\begin{tabular}{|c|c|c|c|c|c|}
\hline Variación Fuente & G L & SC & $\mathrm{CM}$ & Fcal & $\begin{array}{c}\mathrm{F} \\
\mathrm{Tab}\end{array}$ \\
\hline Sum C Total & & 99,97 & & & \\
\hline Tratamiento & 3,00 & 0,67 & 0,22 & 0,08 & 2,87 \\
\hline Exper Error & 36,00 & 99,30 & 2,76 & & \\
\hline Total & 39,00 & 99,97 & 2,98 & & \\
\hline
\end{tabular}

Para realizar el anterior análisis de varianza, se resolvieron cuatro diferentes problemas uno para cada tratamiento. Además, es denotar que en el desarrollo de este artículo se resolvieron diferentes clases de problemas, sus resultados fueron comparados con las soluciones tradicionales (ver tabla 9), sin embargo por razones de espacio solo se ilustra un problema en detalle. Adicionalmente, es importante resaltar que el tiempo de cómputo está directamente relacionado con el número de semestres. Así para un problema de 10 semestres el tiempo promedio de cómputo fue de 371 segundos. En general el tiempo de cómputo promedio para calcular un semestre fue de 39 segundos. Finalmente, con el fin de establecer el mejoramiento en la función fitness logrado por esta metodología, respecto a otras metodologías, se construyó la tabla 9 en la cual se muestra un análisis de 10 diferentes problemas que fueron solucionados considerando 3 salones normales, 2 Talleres y 2 aulas computo. 
Tabla 9. Resumen de problemas analizados.)

\begin{tabular}{|c|c|c|c|c|c|c|}
\hline $\begin{array}{c}\mathrm{N}=\text { Nro. } \\
\text { Semestres }\end{array}$ & $\begin{array}{c}\text { Nro. } \\
\text { Asignaturas }\end{array}$ & $\begin{array}{c}\text { Soluciones }= \\
\text { Factorial (N) }\end{array}$ & $\begin{array}{c}\text { Mejor } \\
\text { Solución }\end{array}$ & $\begin{array}{c}\text { Técnicas } \\
\text { tradicionales }\end{array}$ & Variación & $\begin{array}{c}\text { Tiempo } \\
\text { Computo } \\
(\text { Seg) }\end{array}$ \\
\hline 3 & 17 & $3.56^{\star} 10^{14}$ & 9 & 11 & $18 \%$ & 112 \\
\hline 4 & 23 & $2.59^{\star} 10^{22}$ & 22 & 29 & $24 \%$ & 153 \\
\hline 5 & 29 & $8.84^{\star} 10^{30}$ & 54 & 67 & $20 \%$ & 201 \\
\hline 6 & 35 & $1.03^{\star} 10^{40}$ & 88 & 107 & $18 \%$ & 242 \\
\hline 7 & 43 & $6.04^{\star} 10^{52}$ & 118 & 151 & $22 \%$ & 289 \\
\hline 8 & 48 & $1.24^{*} 10^{61}$ & 145 & 171 & $16 \%$ & 325 \\
\hline 9 & 54 & $2.31^{*} 10^{71}$ & 160 & 191 & $17 \%$ & 345 \\
\hline 10 & 60 & $8.32^{\star} 10^{81}$ & 174 & 220 & $21 \%$ & 371 \\
\hline & Promedio & & & & $19,5 \%$ & \\
\hline
\end{tabular}

Paso 10. Según los resultados de la tabla 9, con la nueva metodología se obtiene un mejoramiento del $19.5 \%$ en el aprovechamiento de los ritmos cognitivos de los estudiantes.

\section{DISCUSION}

Si bien en las diferentes revisiones literarias se pueden encontrar muchas técnicas para la solución de estos problemas, es importante aclarar que en la mismas no existe una heurística basada en algoritmos evolutivos y genéticos la cual trate de optimizar el aprovechamiento de los ritmos cognitivos de los estudiantes, como se ilustro a lo largo de este artículo. Así mismo las restricciones duras y optimización de las suaves son resueltas por el cruce de tres estructuras tridimensionales. De otro lado, las soluciones generadas son bastante consistentes como se ilustra en el análisis de varianza. Finalmente en este punto es importante ilustrar que esta metodología presenta un tiempo de cómputo bastante bajo, el cálculo de una solución solo demora unos pocos segundos.

\section{CONCLUSIONES}

Como conclusiones de este artículo se obtienen: a) se diseña una híper-heurística, basada en la combinación adaptativa de dos técnicas de inteligencia artificial, algoritmos genéticos y algoritmos aleatorios. Estas técnicas evitan los óptimos locales, generando un nuevo padre cada vez que la evolución se estanca en un óptimo local. b) Esta metodología constituye una excelente técnica para resolver esta clase de problema, la cual es por lo menos un 19.5\% más eficiente que las técnicas tradicionales en la resolución de esta clase de problemas. Adicionalmente la codificación de las técnicas inteligentes empleadas, con tres matrices tridimensionales, bajo un enfoque de programación lógica basada en ritmos cognitivos de los estudiantes, representa una nueva metodología no descrita en la literatura. c) La metodología aplicada es fácil de replicar solo requiere una codificación adecuada de la misma descrita en este artículo bajo el enfoque empleado. d) los resultados obtenidos son altamente consistentes como se deduce de los análisis de varianza, encontrándose un modelo no significativo. e) Este tipo de metodologías puede ser aplicado a diferentes clases de problemas como se ilustró en la introducción y en general en la solución de cualquier tipo de problemas NP-duros. f) futuras líneas de investigación integraran más técnicas de inteligencia artificial en esta metodología, buscando una combinación adaptativa y paralela de las mismas con el fin de obtener una mejor solución de este problema, igualmente en estas futuras líneas, será factible asociar funciones de costo, las cuales permitan optimizar los recursos empleados.

\section{AGRADECIMIENTOS}

Este trabajo se desarrolla como parte del proyecto del año sabático del autor del mismo, bajo la financiación de la Universidad Nacional de Colombia. Departamento de Ingeniería Industrial, Manizales - Caldas - Colombia.

\section{REFERENCIAS}

Abbas A. y E. P. K. Tsang, Software engineering aspects of constraint-based timetabling-a case study. Information and Software Technology, 46(6), 359-372 (2004).

Abdul Rahman, S., A. Bargiela, E.K. Burke, E. Özcan, B. McCollum, y P. McMullan, P. Adaptive linear combination of heuristic orderings in constructing examination timetables. European Journal of Operational Research, 232(2), 287-297(2014).

Abdullah, S. y H. Turabieh, On the use of multi neighbourhood structures within a Tabu-based memetic approach to university timetabling problems. Information Sciences, 191, 146-168(2012). 
Abdullah, S., H. Turabieh, B. McCollum, y P. McMullan, A hybrid metaheuristic approach to the university course timetabling problem. Journal of Heuristics, 18(1), 1-23(2010).

Aladag, C. H., G. Hocaoglu, y M.A. Basaran, The effect of neighborhood structures on tabu search algorithm in solving course timetabling problem. Expert Systems with Applications, 36(10), 12349-12356 (2009).

Al-Yakoob, S. M., y H. D. Sherali, A mixed-integer programming approach to a class timetabling problem: $A$ case study with gender policies and traffic considerations. European Journal of Operational Research, 180(3), 1028-1044 (2007).

Asratian, A. S., y D. De. Werra, Discrete Optimization A generalized class - teacher model for some timetabling problems, European Journal Operation Research, 143, 531-542 (2002).

Ayob, M., A. R. Hamdan, S. Abdullah, Z. Othman, M.Z.A. Nazri, K.A. Razak, y N. R. Sabar, (2011). Intelligent Examination Timetabling Software. Procedia - Social and Behavioral Sciences, 18, 600-608 (2011).

Azmi, M. y A.A. Tajudin, A hybrid harmony search for university course timetabling, Multidisciplinary International Conference on Scheduling: Theory and Applications (MISTA 2009), Doblin - Ireland, (August 10-12) 2009.

Berghammer, R., y B. Kehden,. Relation-algebraic specification and solution of special university timetabling problems. The Journal of Logic and Algebraic Programming, 79(8), 722-739 (2010).

Boizumault, P., y Y. Delon, (1996). Constraint logic programming for examination timetabling, The Journal of Logic Programming, 217-233 (1996).

Boland, N., B. D. Hughes, L.T.G. Merlot, y P. J. Stuckey, New integer linear programming approaches for course timetabling. Computers \& Operations Research, 35(7), 2209-2233 (2008).

Burke, E.K., A. J. Eckersley, B. McCollum, S. Petrovic, y R. Qu, Hybrid variable neighbourhood approaches to university exam timetabling. European Journal of Operational Research, 206(1), 46-53 (2010a).

Cangaiovic, M., y J.A.M. Schreuder, Modelling and solving an acyclic multi-period timetabling problem, Discreted Applied Mathematics, 35, 177-195(1992).

Castrillon, O. D. Planning University Schedules Using Evolutionary Algorithms and Cognitive Rhythms, ElIC2013 2nd Electronic International Interdisciplinary Conference 2013, Budapest virtual conference. (2013).

Ceschia, S., L. Di Gaspero, y Schaerf, A. Design, engineering, and experimental analysis of a simulated annealing approach to the post-enrolment course timetabling problem. Computers \& Operations Research, 39(7), 1615-1624 (2012).

Dammak, A., A. Elloumi, y $\mathrm{H}$. Kamoun, Classroom assignment for exam timetabling. Advances in Engineering Software, 37(10), 659-666(2006).

Deris, S., S. Omatu, H. Ohta, y P. Saad, Incorporating constraint propagation in genetic algorithm for university timetable planning. Engineering Applications of Artificial Intelligence, 12(3), 241-253(1999).

Deris, S., S. Omatu, y H. Ohta, Timetable planning using the constraint-based reasoning. Computers \& Operations Research, 27(9), 819-840 (2000).

Franco B. J. F., E. M. Toro, y Gallego R. R. A. Problema de asignación óptima de salones resuelto con Búsqueda Tabú. Ingeniería \& Desarrollo, 149-175 (2008).

Gogos, C., P. Alefragis, y E. Housos, A Multi-staged algorithmic process for the solution of the examination timetabling problem. Annals of Operations Research, 194(1), 203-221(2010).

Granada E., M., E. M. Toro y J. F. Franco B. Programación óptima de horarios de clase usando un algoritmo memético. Scientia et Technica, 255-260 (2006).

Gunadhi, H., V. J. Anand, y Y. Wee Yong, Automated timetabling using an object-oriented scheduler. Expert Systems with Applications, 10(2), 243-256(1996).

Gunawan, A., K-M. Ng, y K.L. Poh, A hybridized Lagrangian relaxation and simulated annealing method for the course timetabling problem. Computers \& Operations Research, 39(12), 3074-3088(2012). 
Hao, J.-K. y U. Benlic, Lower bounds for the ITC-2007 curriculum-based course timetabling problem. European Journal of Operational Research, 212(3), 464-472(2011).

Hernández, R., P. J. Miranda, y P.A. Rey. Programación de horarios de clases y asignación de salas para la facultad de ingeniería de la Universidad Diego Portales mediante un enfoque de programación entera. Revista Ingeniería de Sistemas, 121-141 (2008).

Kahar, M. y G. Kendall. The examination timetabling problem at University Malaysia Pahang: Comparison of a constructive heuristic with an existing software solution, European Journal of Operational Research 207 (2), 557-565 (2010).

Martínez, H., C. Camargo y M. Reyes, Ritmos Cognitivos en la Escuela, Universidad Pedagógica Nacional, Bogotá - Colombia, 2004.

Mausser, H. E., y M. J. Magazine, Comparison of neural and heuristic methods for a timetabling problem. European Journal of Operational Research, 93(2), 271-287(1996).

Mejía, J. y C. Paternina, Asignación de horarios de clases Universitarias Mediante Algoritmos Evolutivos, Educación e Ingeniería 5 (9), 140 - 149 (2010).

Naji Azimi, Z, Hybrid heuristics for Examination Timetabling problem. Applied Mathematics and Computation, 163(2), 705-733(2005).

Pongcharoen, P., W. Promtet, P. Yenradee, y C. Hicks, Stochastic Optimization Timetabling Tool for university course scheduling. International Journal of Production Economics, 112(2), 903-918(2008).

Qaurooni, D., y M. R. Akbarzadeh-T, Course timetabling using evolutionary operators. Applied Soft Computing, 13(5), 2504-2514(2013).

Smith, K. A., D. Abramson. y D. Duke, Hopfield neural networks for timetabling: formulations, methods, and comparative results. Computers \& Industrial Engineering, 44(2), 283-305(2003).

Suarez, Víctor. Asignación de horarios de clase en instituciones de educación básica y media de acuerdo a ritmos de eficiencia cognitiva. Tesis de Maestría. Universidad Nacional de Colombia. (2012).

Suarez, V. y O. Castrillón. Diseño de una metodología basada en técnicas inteligentes para la distribución de procesos académicos en ambientes de trabajo Job Shop, XV Congreso de Ingeniería de la Organización Universidad Politécnica de Cartagena, Cartagena - España: 285 - 289 (2011).

Suarez, V., A. Guerrero. Y O. Castrillón, Programación de Horarios Escolares basados en Ritmos Cognitivos usando un Algoritmo Genético de Clasificación No-dominada, NSGA-II, Información Tecnológica 24 (1), 103 - 113(2013a).

Suarez, V., O. Castrillón y A. Guerrero, Asignación de horarios de clase basado en los ritmos de aprendizaje de los estudiantes usando un algoritmo genético, Ingeniería y Ciencia 9 (17), 77- 95(2013b).

Thepphakorn, T., P. Pongcharoen, y C. Hicks, An ant colony based timetabling tool. International Journal of Production Economics, 1-14(2013).

Thompson, J. M. y K. Dowsland, A robust simulated annealing based examination timetabling system. Computers \& Operations Research, 25(7-8), 637-648(1998).

Valentinuzzi, V. S. y A. J. Fontenele, Los ritmos biológicos y el aprendizaje, Revista Ingenierías, XI(40), 41-46(2008).

Wu, C.-C. Parallelizing a CLIPS-based course timetabling expert system. Expert Systems with Applications, 38(6), 7517-7525(2011). 